\title{
5. Der spezifisch diskurstheoretische Zugang
}

Gemeinsam ist allen fünf vorgestellten Projekten, dass sie in Form von selbst produzierten Medien eigene, alternative Öffentlichkeiten herstellen. Projektinterne Medien wie zum Beispiel Broschüren, Flyer, Plakate, Pressemitteilungen, Stellungnahmen und eigene Zeitschriften spielen für die Öffentlichkeits- und Bildungsarbeit der Einrichtungen eine zentrale Rolle. Es werden dort aber auch Erfahrungen und politische Positionen ausgetauscht und diskutiert. Diese von den Projekten produzierten Text- und Bildmaterialien sollen im Zentrum der empirischen Untersuchung der vorliegenden Arbeit stehen und in Anlehnung an Foucault diskursanalytisch aufgearbeitet werden.

\subsection{Warum Foucault?}

Der Aids-Aktivist Douglas Crimp erzählt in einem Interview, dass beinahe alle »Act Up«Aktivist"innen eine Ausgabe von »Sexualität und Wahrheit 1« in der Tasche gehabt hätten. ${ }^{1}$ Diese sicher etwas übertriebene Darstellung veranschaulicht, wie sich Foucaults diskurstheoretisches Denken in den queeren Aktivismus und die gleichzeitig oder im Anschluss daran entstandene queere Theoriebildung eingeschrieben hat. Foucault, der selbst acht Jahre nach dem Erscheinen des ersten Bandes von "Sexualität und Wahrheit 1 « - und ohne die Zeit zu haben, den letzten Band zu veröffentlichen - an Aids gestorben ist, hat mit seinen Arbeiten die bis dahin gängige Vorstellung von sexueller Befreiung auf den Kopf gestellt und damit auch den queeren Aids-Aktivismus mitgeprägt. Für die Aktivist*innen war es mit Foucault nicht mehr möglich, eine Praxis, in der mensch einfach Ja zu Sexualität sagt, als »Befreiung « zu verstehen. Foucault kritisiert in »Sexualität und Wahrheit 1« eine einfache Opposition zwischen »Unterdrückung« und "Befreiung ${ }^{2}{ }^{2}$ Sexualität ist, seinen Ausführungen zufolge, nicht etwas, das im Sinne eines einfachen Machtverständnisses unterdrückt und verboten wird. Im Gegenteil: Es hat »in modernen industriellen Gesellschaften« eine »Explosion« von »machtvollen Diskursen« über den Sex gegeben: 
»Die vielfältigen Sexualitäten - diejenigen, die auf verschiedenen Altersstufen auftreten (Sexualitäten des Säuglings oder des Kindes), diejenigen, die sich an Neigungen und Praktiken heften (Sexualität des Homosexuellen, des Cerontophilen, des Fetischisten ...), diejenigen, die in diffuser Weise Beziehungen besetzen (Sexualität der Arzt-Patient-Beziehung, der Lehrer-Schüler-oder der Psychiater-Irrer-Beziehung), diejenigen, die in bestimmten Räumen ihr Unwesen treiben (Sexualität des Heimes, der Schule, des Cefängnisses) - sie alle bilden das Korrelat präziser Machtprozeduren. «³

Diese machtvollen Diskurse über den Sex durchdringen die Körper und das Verhalten - und konstituieren Subjekte. ${ }^{4}$ Das Subjekt, das sich von der Repression der Sexualität befreien möchte, indem es Ja sagt zum Sex, folgt erst recht dem »Lauf des Sexualitätsdispositivs«, weil es Ja sagt zu den machtvollen Diskursen, die es konstituieren: »Glauben wir nicht, daß man zur Macht `nein sagt, indem man zum Sex ja sagt; man folgt damit vielmehr dem Lauf des allgemeinen Sexualitätsdispositivs. [...] Ironie dieses Dispositivs: es macht uns glauben, daß es darin um unsere >Befreiung ‘ geht. $\aleph^{5}$ Realpolitisch haben die »Act Up«-Aktivist*innen im Sinne Foucaults versucht, in jene machtvollen Diskurse zu intervenieren, die festlegen wollten, wer die Homosexuellen sind. Dabei ging es zu Zeiten der Aids-Krise vor allem darum, die Verknüpfung von Homosexualität und Krankheit zu entkoppeln.

Queere Theoriebildungen haben an vielen Stellen an Foucaults Denken angeschlossen. So ist beispielsweise die prominent von Judith Butler geübte Kritik an der Kategorie »Frau als Subjekt des Feminismus« eng an ein Denken Foucaults geknüpft, mit dem gezeigt werden kann, dass Diskurse, die als Machttechniken fungieren, die "Frau«, die als feministisches Subjekt der Befreiung gilt, selbst hervorbringen. ${ }^{6}$ Damit begründete Foucault ein für queere Theorie und Praxis zentrales Denken mit, demzufolge Widerstand heißt, immer wieder neu zu verhandeln, wer mensch sein möchte: „Das Hauptziel besteht heute zweifellos nicht darin, herauszufinden oder abzulehnen, was wir sind. Wir müssen uns vorstellen und konstruieren, was wir sein könnten, wenn wir uns dem doppelten politischen Zwang entziehen wollen, der in der gleichzeitigen Individualisierung und Totalisierung der modernen Machtstrukturen liegt. $\aleph^{7}$ Für Foucault ist Widerstand gleichzeitig Ausgangspunkt und analytische Haltung ${ }^{8}$ seiner Analyse von Machtverhältnissen. Um Machtverhältnisse zu erfassen, ist es ihm zufolge sinnvoll, vom Widerstand gegen ebendiese Machtverhältnisse ausgehen:

»Ich schlage daher vor, zum Ausgangspunkt eine Reihe von Widerständen zu nehmen, die sich in den letzten Jahren entwickelt haben: den Widerstand gegen die Macht der

\footnotetext{
3 Ebd., S. 51.

4 Ebd., S. 73.

5 Ebd., S. $151 \mathrm{ff}$.

6 J. Butler: Das Unbehagen der Ceschlechter, S. $16 \mathrm{f}$.

7 Michel Foucault: »Das Subjekt und die Macht«, in: Hubert L. Dreyfus/Paul Rabinow (Hg.), Michel Foucault. Jenseits von Strukturalismus und Hermeneutik, Frankfurt a.M.: Athenäum 1987, S. 269294, hier S. 280.

8 Vom Widerstand ausgehen heißt, der Analyse eine (widerständige) Haltung zugrunde zu legen, die letztendlich darauf abzielt, nicht dermaßen regiert zu werden.
} 
Männer über die Frauen, der Eltern über die Kinder, der Psychiatrie über die Ceisteskranken, der Medizin über die Bevölkerung, der staatlichen Verwaltung über die Lebensweise der Menschen. «?

Foucault nennt typische Merkmale der Widerstände, die er zum Ausgangspunkt der Analyse von Machtverhältnissen machen will: Es sind Widerstände gegen das, was »die Einzelnen « an ihre »Identität bindet «, Widerstände, die in Frage stellen, auf welche »Art und Weise, wie Wissen zirkuliert und funktioniert«, und Widerstände, die sich um die Frage drehen $»$ Wer sind Wir? ${ }^{10}$ Nicht nur weil queere Theorie und Praxis immer schon durch Foucault hindurch geht, sondern auch weil die Forschungsfrage der vorliegenden Untersuchung danach, wer diese queeren politischen Subjekte, die sich nicht an eine (eindeutige) Identität binden lassen, sind (sein wollen), von Foucaults Denken ausgeht, ist ein diskurstheoretischer Zugang gewählt worden.

\subsection{Ein Versuch, den "Meister" selbst sprechen zu lassen}

Was ist aber genau das Foucault'sche Programm und worin unterscheidet es sich von anderen Fragen? In der Vorlesung »Subjektivität und Wahrheit« resümiert Foucault, dass es ihm im Unterschied zur klassischen Philosophie weder um die Frage gehe, ob es für das Subjekt überhaupt eine Möglichkeit der Wahrheit gibt, noch darum, nach den Möglichkeiten und Voraussetzungen zu fragen, »wahr« über das Subjekt zu sprechen. Das, was ihn interessiert, ist die »historisch-philosophische« Frage, »welche Auswirkungen die Existenz eines Diskurses, der den Anspruch erhebt, über die Subjektivität die Wahrheit zu sagen, auf diese hat. ${ }^{11}$ Um diese Frage drehen sich auch seine Arbeiten zum Wahnsinn, zur Krankheit und zum Verbrechen. Er versucht aufzuzeigen, wie sich bestimmte Praktiken herausgebildet haben, die einen

»[...] wahren Diskurs über den kranken Körper oder den kriminellen Charakter implizieren, und wie der Bezug, den wir zu uns selbst haben, [...] von der Existenz dieses wahren Diskurses und den Wirkungen, die er hervorruft, von den Pflichten, die er auferlegt, und den Versprechungen, die er andeutet oder formuliert, affiziert, modifiziert, transformiert, strukturiert wird. (2 $^{12}$

Dabei verortet Foucault sein Programm in »der« Philosophie, der es als Diskurs gemeinsam sei, die Frage nach der aletheia (Wahrheit), der politeia (Macht) und dem ethos miteinander zu verbinden. In der Art und Weise, diese drei Ebenen zu verbinden, grenzt er sich von einem prophetischen Diskurs der »verheißenden Versöhnung« ab, genauso wie von einem philosophischen Diskurs der Weisheit, der »die grundlegende Einheit der Wahrheit, der Macht und des ethos zu denken und zu sagen versucht « ${ }^{13}$ Er grenzt

9 M. Foucault: Das Subjekt und die Macht, S. 273.

10 Ebd., S. $273 \mathrm{ff}$.

11 M. Foucault: Subjektivität und Wahrheit, S. 28.

12 Ebd., S. 29.

13 Michel Foucault: Der Mut zur Wahrheit. Vorlesung am Collège de France 1983-1984, Berlin: Suhrkamp Verlag 2010, S. 98. 
sich aber auch von einem philosophischen »Diskurs der Heterogenität und der Trennung von aletheia, politeia und ethos « $a b$, den er den »technischen « Diskurs nennt. ${ }^{14}$ Entgegen der Diskurse der verheißenden Versöhnung, der Einheit und der Heterogenität, verortet Foucault seine Analysen in einem »parrhesiastischen« Diskurs:

»Das ist die parrhesiastische Einstellung, jene die eben hartnäckig und immer wieder von neuem beginnend versucht, die Frage nach der Wahrheit mit der Frage nach ihren politischen Bedingungen und nach ihren ethischen Differenzierungen, die den Zugang zu ihr eröffnet, zu verknüpfen; die stets und immer die Frage nach der Macht mit der Frage nach ihrem Verhältnis zur Wahrheit und zum Wissen einerseits und mit der ethischen Differenzierung andererseits verknüpft; die schließlich unablässig das moralische Subjekt mit der Frage nach dem wahren Diskurs, durch den sich dieses moralische Subjekt konstituiert, und mit den Machtverhältnissen, in denen sich dieses Subjekt bildet, verknüpft. « ${ }^{15}$

\section{Diskurs und Wahrheit}

Was versteht Foucault nun aber unter Diskurs, was unter Subjekt und Macht? Und wie sind diese Begriffe miteinander verknüpft? Das, was Foucault in seinen Analysen betrachtet, sind zumeist Diskurse - oder auch Dispositive - in ihrer Verknüpfung mit Machtverhältnissen, die wiederum Subjekte hervorbringen. Je nach Untersuchungsgegenstand (z.B. Wahnsinn, die Strafgesellschaft, der Neoliberalismus, Subjektivität und Wahrheit, Sexualität) und Frage analysiert er beispielsweise Gesetzestexte, Krankenakten, Theaterstücke, philosophische Texte, aber auch architektonische Anordnungen, um Kontinuitäten und Brüche herauszuarbeiten und um zu verstehen, welche Subjekte sie hervorbringen, welche Rolle sie im Spiel der Macht haben und auf welche Notwendigkeiten sie antworten. Er spricht von Diskursen - also ein Ensemble von (diskursiven) Praktiken ${ }^{16}$ - die den Gegenstand, von dem sie sprechen, hervorbringen, ${ }^{17}$ aber auch von Diskursen als "Gesamtheit erzwungener und erzwingender Bedeutungen, die die gesellschaftlichen Verhältnisse durchziehen «. ${ }^{18}$ In seiner Antrittsvorlesung am Collège der France erklärt Foucault, dass der Diskurs von äußeren Ausschlussmechanismen und inneren Ordnungen und Kontrollmechanismen bestimmt sei. Die inneren Ordnungen

14

$$
\text { Ind }
$$

In der deutschsprachigen Diskursforschung wird häufig versucht, zwischen diskursiven (zumeist ritualisierten) Praktiken und nicht diskursiven Praktiken zu unterschieden. Vgl. Silke van Dyk: Verknüpfte Welt oder Foucault meets Latour. Zum Dispositiv als Assoziation«, in: Robert Feustel/Maximilian Schochow (Hg.), Zwischen Sprachspiel und Methode. Perspektiven der Diskursanalyse, Bielefeld: transcript Verlag 2014, S. 169-196. Foucault selbst sagt in einem Interview, in dem es um den Dispositivbegriff geht, dass die Unterscheidung diskursiv/nicht diskursiv nicht so relevant sei: »für das, was ich mit dem Dispositiv sagen will, ist es kaum von Bedeutung, zu sagen: das hier ist diskursiv und das nicht. «Michel Foucault: Dispositive der Macht. Über Sexualität, Wissen und Wahrheit, Berlin: Merve Verlag 1978, S. 125.

17 Michel Foucault: Archäologie des Wissens, Frankfurt a.M.: Suhrkamp Verlag 2015 [1981], S. 74.

18 Michel Foucault: Geometrie des Verfahrens. Schriften zur Methode, Frankfurt a.M.: Suhrkamp Verlag 2009, S. 213. 
und Kontrollmechanismen seien die Anordnungen, Klassifikationen und Verteilungsprinzipien, also Regeln des Diskurses, die Kontrolle ausüben. Die äußeren Ausschlussmechanismen des Diskurses sind verknüpft mit der Macht und dem Begehren und beziehen sich in unserer Zeit vornehmlich auf den Willen zur Wahrheit, aber auch auf den Ausschluss des Wahnsinns und das Verbot: »Drei große Ausschließungssysteme treffen den Diskurs: das verbotene Wort; die Ausgrenzung des Wahnsinns; der Wille zur Wahrheit. [...] Während die beiden ersten immer schwächer werden, und ungewisser, sofern sie vom Willen zur Wahrheit durchkreuzt werden, wird dieser immer stärker, immer tiefer, immer unausweichlicher. $\ll^{19}$

Der »Wille zur Wahrheit« ist ein Ausschlussmechanismus, der von außen auf den Diskurs wirkt und seit Platon unsere Diskurse durchzieht. Während im Fall von Wahnsinn und Verbrechen die Wahrheit von außen kommt und wahnsinnige oder delinquente Subjekte konstituiert, ist der Bezug zur Wahrheit in den Diskursen um den Sex im Gegensatz dazu um sich selbst herum angeordnet. Der »wahre Diskurs« im Bereich der Sexualität ist ein Diskurs des Subjekts über sich selbst, der mit dem Christentum $\mathrm{zu}$ einer »Geständnispraxis« geworden ist, der eine Schuld vorgängig ist. Wenn Foucault über die Beziehung zwischen Subjektivität und Wahrheit spricht, denkt er kein diesen Diskursen vorgängiges Subjekt, sondern ein Subjekt, das sich in Beziehung zur Wahrheit herausbildet. Die Wahrheit ist etwas Bindendes, mittels dessen sich Subjekte konstituieren. ${ }^{20}$ Der Diskurs produziert also Wahrheiten, die die Subjekte an sich binden. Dabei ist das spezifische Verhältnis zwischen Subjektivität und Wahrheit selbst auch diskursiv hervorgebracht. Diskurse stehen aber nicht nur in einem speziellen Verhältnis zur Wahrheit, sondern auch zur Macht.

\section{Die Macht}

Der Diskurs ist gleichzeitig »eine Waffe der Macht«, »ein strategisches Feld der Kontrolle, der Unterwerfung «, ${ }^{21}$ aber auch »Hindernis, Gegenlager, Widerstandspunkt und Ausgangspunkt für eine entgegengesetzte Strategie«. ${ }^{22}$ Er ist das, »worum grundsätzlich ein Kampf geführt wird «. ${ }^{23}$ Um Subjektivierungsformen zu untersuchen, gilt es also auch, das Wechselspiel zwischen Diskurs und Macht zu rekonstruieren, also der Frage nachzugehen, inwiefern der Diskurs Macht »befördert « und wo er sie »zerbrechlich« macht. ${ }^{24}$ Foucault grenzt seinen Machtbegriff immer wieder von anderen Macht-Vorstellungen $\mathrm{ab}$. Er wendet sich gegen einen repressiven Machtbegriff, aber auch gegen einen rein juridischen oder souveränen Machtbegriff oder die Vorstellung, dass Macht etwas sei, das eine Gruppe besitzt. Das sind, so Foucault, alles Endpunkte der Macht. Unter Macht versteht er dementgegen »die Vielfältigkeit von Kräfteverhältnissen, die ein Gebiet bevölkern und organisieren, das Spiel, das in

19 Michel Foucault: Die Ordnung des Diskurses, Frankfurt a.M.: Fischer Taschenbuch Verlag 2017, S. 16.

20 M. Foucault: Subjektivität und Wahrheit, S. $29 f$.

21 M. Foucault: Ceometrie des Verfahrens, S. 213.

22 M. Foucault: Der Wille zum Wissen, S. 100.

23 M. Foucault: Ceometrie des Verfahrens, S. 213.

24 M. Foucault: Der Wille zum Wissen, S. 100. 
unaufhörlichen Kämpfen und Auseinandersetzungen diese Kräfteverhältnisse verwandelt, verstärkt, verkehrt«. ${ }^{25}$ Das Spiel dieser Kräfteverhältnisse ist »erkennbar« und damit auch analysierbar, weil sie von einem »Kalkül« durchsetzt sind: »Die Macht ist der Name, den man einer komplexen strategischen Situation in einer Gesellschaft gibt.«Sie ist durchdrungen von einer »Rationalität von Taktiken« und Strategien, die es herauszuarbeiten gilt. ${ }^{26}$

Foucault unterscheidet seinen Machtbegriff auch explizit vom Gewalt-Begriff. Gewalt ist im Gegensatz zur Macht unmittelbar auf "Körper und Dinge ausgerichtet«, sie zerbricht und »schneidet « alle Möglichkeiten ab. Konsens und Gewalt können zwar »Mittel und Prinzip« der Macht sein, sie sind aber nicht ihr »Wesen«. Macht ist etwas, das im Unterschied zur Gewalt auf Handlungen ausgerichtet ist und Spielräume/Möglichkeitsräume eröffnet. Entsprechend ist da, wo die Macht ist, auch Widerstand. ${ }^{27}$ In seiner Vorlesung zur »Strafgesellschaft« erklärt Foucault beispielsweise, inwiefern sich der Bürgerkrieg »auf der Bühne der Macht « abspielt: ${ }^{28}$ Im Bürgerkrieg wird versucht Fragmente und Symbole der Macht zu aktivieren, sie anzueignen und umzukehren, wodurch der Mythos der Macht bestätigt wird, der nach einem »erdichteten Anführer« als Repräsentant verlangt: »Es gibt keinen Bürgerkrieg ohne Arbeit der Macht, Arbeit an der Macht. ${ }^{29}$ Gleichzeitig zeigt Foucault auf, wie der Bürgerkrieg auch das ist, was »die Macht umtreibt «. ${ }^{30}$ Widerstand - und das erklärt auch, warum er, Foucault zufolge, ein guter Ausgangspunkt für die Analyse von Machtverhältnissen ist - ist konstitutiv verwoben mit der Macht. Letztlich geht es Foucault, wie er selbst resümiert, darum, Subjektivierungsformen zu untersuchen, die durch Regime von Macht-Wissen hervorgebracht werden.

\section{Foucaults Vorlesungen als Werkzeugkasten}

Foucault selbst hat nie eine eigene Methodologie entwickelt, will aber seine Arbeiten als »Werkzeugkasten « verstanden wissen, aus dem mensch sich bedienen kann. ${ }^{31}$ In der »Archäologie des Wissens« hat er zwar versucht, die methodischen Implikationen seiner früheren Arbeiten »Wahnsinn und Gesellschaft«, »Die Geburt der Klinik« und »Die Ordnung der Dinge« einzuordnen und kritisch zu reflektieren. Diese Reflexionen beziehen sich aber nur auf eben genannte frühere Arbeiten beziehungsweise beschreibt er sie selbst im Nachhinein als einen »blinden Versuch«, so etwas wie eine methodische Standortbestimmung zu leisten. ${ }^{32}$ Allerdings stellt Foucault auch in seinen anderen Arbeiten, insbesondere in seinen Vorlesungen, immer wieder methodisch wertvolle

25 Ebd., S. 93.

26 Ebd., S. 95.

27 M. Foucault: Das Subjekt und die Macht, S. $285 f$.

28 M. Foucault: Die Strafgesellschaft, S. 50.

29 Ebd., S. 53.

30 Er bezieht sich hier vor allem auf die Aufstände der Nu-Pieds, die er ausführlicher analysiert hat.

31 Siegfried Jäger: Kritische Diskursanalyse. Eine Einführung, Münster: Unrast Verlag 2015, S. 19.

32 Clemens Kammler: »Archäologie des Wissens«, in: Clemens Kammler/Rolf Parr/Ulrich ]. Schneider et al. (Hg.), Foucault-Handbuch. Leben - Werk - Wirkung, Stuttgart, Weimar: Verlag J.B. Metzler 2014, S. 51-62. 
Reflexionen an. Es können aber auch aus seinen Analysen selbst - aus der Art und Weise, wie er vorgeht und arbeitet - methodische Implikationen herausgelesen werden. So haben insbesondere seine Vorlesungen die vorliegende Analyse beeinflusst. Die methodischen Reflexionen sind, genauso wie Foucaults konkrete Vorgehensweise in den Analysen der Vorlesungen, vor dem Hintergrund der Methode der Kritischen Diskursanalyse von Siegfried Jäger mit dem Ziel, zu verstehen, wie Foucault selbst methodisch vorgeht, gelesen worden. Beides - die Kritische Diskursanalyse von Jäger und das durch die Vorlesungen angeeignete Verständnis - fließt in die methodische Vorgehensweise der vorliegenden Arbeit ein. Allerdings ist es schwierig, die Bezüge zu Foucaults Vorlesungen zu explizieren. Am Beispiel einiger methodischer Reflexionen, die Foucault vor allem in der Vorlesung »Hermeneutik des Subjekts« angestellt hat, soll versucht werden zumindest einige Aspekte, die die Vorlesungen Foucaults als »Werkzeugkasten« zu bieten haben, zu benennen.

Zunächst einmal reflektiert Foucault oft zu Beginn eines Vorlesungszyklus das Verhältnis zwischen dem aktuellen und dem vorher behandelten Thema und zeigt dabei auf, auf welcher Ebene sich seine Analyse bewegt. So beschreibt er den Unterschied zwischen seinem Forschungsinteresse in den Vorlesungen zu »Subjektivität und Wahrheit« und in jenen zur »Hermeneutik des Subjekts« folgendermaßen:

»Dieses Jahr möchte ich mich von diesem Beispiel und dem Material der aphrodisia und der sexuellen Verhaltensordnung etwas entfernen und die darin enthaltene allgemeinere Problematik von `Subjekt und Wahrheit< herausarbeiten. Cenauer gesagt, ich möchte auf keinen Fall die historische Dimension, in die ich die Problematik von Subjektivität und Wahrheit einzubetten versucht habe, ausschalten oder auslöschen, ich möchte sie aber in viel allgemeinerer Gestalt erscheinen lassen.«33

Während es also zunächst noch um die Beziehung zwischen Subjektivität und Wahrheit in den Diskursen der Sexualität ging, geht es ihm in der »Hermeneutik des Subjekts« allgemeiner um das Verhältnis zwischen Subjektivität und Wahrheit. In seinen Vorlesungen macht Foucault auch zumeist in Abgrenzung zu »üblichen« Fragestellungen und Themen deutlich, welche Fragen seine Analyse durchziehen. So benennt er für die »Hermeneutik des Subjekts« folgende Frage, die die Analyse von Subjektivität und Wahrheit leiten soll: » $\mathrm{Zu}$ welcher geschichtlichen Gestalt haben sich im Abendland die Beziehungen dieser beiden Elemente, Subjekt und Wahrheit, die üblicherweise nicht der Gegenstand historischer Praxis oder Analyse sind, verknüpft? « ${ }^{34}$ Weiters expliziert er genau und oft auch über mehrere Vorlesungen hinweg, was er analysiert, und begründet es. Der konkrete Gegenstand in der »Hermeneutik des Subjekts« ist entsprechend die »Sorge um sich «, die er als einen »wichtigen Leitfaden« versteht, um die Geschichte der »Subjektivierungspraktiken « zu untersuchen. ${ }^{35}$ Die Diskurse zur »Sorge um sich « will er sich in drei bestimmten Zeiträumen anschauen, indem er besonders auf Brüche und Kontinuitäten achtet: 
»Dieses Jahr [...] werde ich versuchen drei Momente zu isolieren, die mir interessant erscheinen: den sokratisch-platonischen Moment, das Auftauchen der epimeleia heauton in der philosophischen Reflexion, zweitens, die Periode des Coldenen Zeitalters der Selbstkultur [culture de soi], der Sorge um sich selbst, die in den ersten zwei nachchristlichen Jahrhunderten anzusiedeln wäre, und drittens, den Übergang vom 4. zum 5. Jahrhundert, d.h. grob gesprochen den Übergang von der heidnischphilosophischen Askese zur christlichen. « ${ }^{36}$

Hier wird bereits deutlich, dass dieser Absteckung des Untersuchungszeitraums ein ausführliches Wissen vorausgeht, von dem ausgehend er bestimmen kann, in welchen Zeiträumen es Verschiebungen oder Neuordnungen gegeben hat oder welche Zeiträume besonders repräsentativ das vorgegebene Thema behandeln (»Das Goldene Zeitalter der Selbstkultur«). Aber auch der Entscheidung, sich mit der »Sorge um sich« zu beschäftigen und nicht mit dem »Erkenne dich selbst «, das weitaus bekannter ist als ersteres, geht ein tiefes Wissen um die Debatten und Diskurse im alten Griechenland und Rom voraus. ${ }^{37}$ Nachdem der Gegenstand und der Zeitraum abgesteckt sind, in dem sich Foucault der Beziehung zwischen Subjektivität und Wahrheit nähern möchte, wendet er sich der konkreten Analyse von Texten zu, die er für repräsentativ hält. Auch hier begründet er die Auswahl des Textes (Alkibiades), den er genauer analysiert: »Wir haben hier die erste, und wir können sogar sagen: (unter) allen Platon-Texten haben wir hier die einzige umfassende Theorie des Selbst. Wir können das als den ersten großen theoretischen Auftritt der epimeleia heauton ${ }^{38}$ betrachten. ${ }^{39}$ Die Analyse Foucaults umfasst neben einer detaillierten Auseinandersetzung mit dem, wie im Text die Beziehung zwischen Subjektivität und Wahrheit verhandelt wird, auch eine Einordnung des diskursiven Kontexts des Themas: „Es gab, wenn Sie es so wollen, lange vor Platon, lange vor dem Text Alkibiades und lange vor Sokrates eine regelrechte Technologie des Selbst, die im Zusammenhang mit dem Wissen stand, sei es mit bestimmten Kenntnissen, sei es mit dem Zugang zur Wahrheit überhaupt. « ${ }^{40}$

In diesen Analysen Foucaults wird bereits ersichtlich, woran Jäger anschließt, wenn er eine Methode der Kritischen Diskursanalyse in Anlehnung an Foucault entwickelt. Was Foucault hier macht, ist - ähnlich, wie Jäger es auf ihn rekurrierend vorschlägt erstens, in Bezug auf die Fragestellung, den Gegenstand einzugrenzen, ausgehend von einer umfangreichen Kenntnis des Materials, die unter anderem durch die Strukturanalyse erlangt wird; und zweitens, für die Fragestellung repräsentative Diskursfragmente auszuwählen. Auch in den detaillierten Analysen einzelner repräsentativer Texte (z.B. Alkibiades, ${ }^{41}$ König Ödipus ${ }^{42}$ oder die Traumdeutung Artemidors ${ }^{43}$ ) lässt sich

42 Michel Foucault: Die Regierung der Lebenden. Vorlesung am Collège de France 1979-1980, Berlin: Suhrkamp Verlag 2014. 
erkennen, wie Foucault vorgeht. In seiner detaillierten Analyse solcher Texte arbeitet er auf sprachlicher Ebene beispielsweise die Metapher der "Schifffahrt « ${ }^{44}$ heraus oder überlegt, welche Bedeutung der Wurzel mel des Begriffs epimeleia (Sorge) zukommt, um aufzuzeigen, welche Wahrheiten wie hergestellt werden. ${ }^{45}$ Es ist also möglich, aus der Auseinandersetzung mit Foucaults Vorlesungen eine Idee davon zu bekommen, welche Fragen eine Diskursanalyse stellt, wie sie ihren Gegenstand umreißt, wie Material begründet ausgewählt wird und wie eine detaillierte Analyse aussehen kann. Gleichzeitig wird deutlich, was schon vielfach gesagt wurde, nämlich dass es »die« FOUCAULT'sche Diskurstheorie nicht gibt. ${ }^{46}$ Neben dem durch die Vorlesungen Foucaults erarbeiteten, oft intuitiven Verständnis ist vor allem das methodische Instrumentarium, welches Siegfried Jäger 1993 erstmals entwickelte, Grundlage für das methodische Vorgehen der vorliegenden Untersuchung.

\subsection{Von Foucault zur Kritischen Diskursanalyse (KDA)}

Jäger gilt als einer der ersten, der im deutschsprachigen Raum ein konkretes »Methodenbuch « einer Diskursanalyse, die an Foucault orientiert ist, entwickelt hat. Er selbst verfolgte zunächst einen ideologiekritischen und sprachwissenschaftlichen Zugang und hat vor allem über Jürgen Link den Wert der Diskurstheorie für die Analyse rechter Diskurse - mit denen er sich damals beschäftigte - erkannt. ${ }^{47}$ Entsprechend hat Jäger in seinem Versuch, so etwas wie eine "angewandte« Diskurstheorie zu entwickeln, auch über Foucault hinausgearbeitet. Zum einen, indem er Elemente von Jürgen Link, insbesondere die Kollektivsymbolik, übernommen hat, zum anderen, indem er zumindest in seinen früheren Einführungen mit Bezug auf Alexei Nikolajewitsch Leontjew versucht hat, das Verhältnis zwischen Subjekt und Diskurs näher zu fassen. Jäger hat aber auch Überlegungen aus der Argumentationstheorie, der Textlinguistik sowie der Psycho- und Soziolinguistik integriert. ${ }^{48} 2012$ - also fast zwanzig Jahre nachdem sein erstes Methodenbuch erschienen ist - hat Jäger dann eine vollständig überarbeitete Ausgabe der Kritischen Diskursanalyse herausgebracht, in der er auf die Tätigkeitstheorie von Leontjew verzichtet und stattdessen die Diskursanalyse um die Dispositivanalyse erweitert. ${ }^{49}$ Die wohl bekannteste und fast alle seine Arbeiten durchziehende Definition des Diskurses von Jäger ist die vom »Fluss von sozialen Wissensvorräten durch die Zeit «. ${ }^{50}$

47

\section{Foucault: Der Mut zur Wahrheit, S. $158 \mathrm{f}$.}

Ebd., S. 26. Qualitative Sozialforschung 7 (2006), 89 Absätze.

Rainer Diaz-Bone: »Kritische Diskursanalyse: Zur Ausarbeitung einer problembezogenen Diskursanalyse im Anschluss an Foucault. Siegfried Jäger im Gespräch mit Rainer Diaz-Bone«, in: Forum

]äger hofft, mit Hilfe von Foucault einen ideologiekritischen Zugang überwinden zu können. R. Diaz-Bone: Kritische Diskursanalyse: Zur Ausarbeitung einer problembezogenen Diskursanalyse im Anschluss an Foucault. Siegfried Jäger im Gespräch mit Rainer Diaz-Bone.

Er selbst sagt, die Tätigkeitstheorie von Leontjew sei nicht falsch gewesen, aber die Lücke, die er mit Leontjew zu schließen versucht habe, könne auch mit dem Dispositivbegriff von Foucault geschlossen werden. S. Jäger: Kritische Diskursanalyse, S. 37. 
Diese Definition hat er mehrmals abgewandelt und erweitert, auch weil sie von Jürgen Link als präzise, aber zu »linear « kritisiert wurde. ${ }^{51}$ In der Auflage aus dem Jahr 2015 definiert Jäger, sich selbst und Margarete Jäger zitierend, Diskurs als einen

$»[$...] rhyzomartig verzweigte[n] mäandernde[n] $>$ Fluss $<$ von $>$ Wissen $<$ bzw. sozialen Wissensvorräten durch die Zeit<, der durchaus auch einmal rückwärts fließen kann, und [der ...] die Vorgaben für die Subjektbildung und die Strukturierung und Cestaltung von Cesellschaften [schafft], die sich entsprechend als außerordentlich vielgestaltig erweisen. ${ }^{52}$

In der neuen Auflage erweitert Jäger sein Diskursverständnis zudem um raumtheoretische Überlegungen und spricht entsprechend vom Fluss von »Wissen durch Raum und Zeit«. Entgegen mancher empirischen Missverständnisse, ${ }^{53}$ versteht Jäger unter Diskursen nicht nur textuelle, sprachliche Praktiken, sondern auch nichtsprachliche Praktiken. ${ }^{54}$ Auch Jäger betont die Verknüpfung von Diskursen oder Wissen mit der Macht: "Sie [Diskurse] tragen dabei zur Strukturierung von Macht- und Herrschaftsverhältnissen in der Gesellschaft bei. ${ }^{55}$ Wie aber sieht die methodische Vorgehensweise aus, die Jäger in Anlehnung an Foucaults Werkzeugkiste entwickelt hat? Die konkrete Herangehensweise, die Jäger vorschlägt, besteht aus fünf Hauptphasen: erstens die Konzeptionsphase, zweitens die Erhebungsphase (Erschließung und Aufbereitung der Materialbasis, des Korpus), drittens die Strukturanalyse, viertens die Feinanalyse und fünftens die zusammenfassende Interpretation. ${ }^{56}$ Diese fünf Phasen werden im Folgenden, Bezug nehmend auf die konkrete Umsetzung, erläutert.

In der ersten Konzeptionsphase geht es zunächst einmal darum, den »Gegenstand der Untersuchung « »sorgfältig « zu benennen und zu begründen. ${ }^{57} \mathrm{Um}$ das Untersuchungsziel und den Untersuchungsgegenstand einzuordnen und einzugrenzen, ist das von Jäger geschaffene begriffliche Instrumentarium hilfreich. Das, was in einer Arbeit analysiert werden kann, sind niemals ganze Diskurse, sondern immer Diskursstränge - also Diskurse zu einem bestimmten Thema auf verschiedenen Ebenen, die sich zu einem Strang bündeln. In dieser Arbeit wurden analog zu diesen Überlegungen queere Diskurse auf aktivistischer Ebene von fünf ausgewählten Projekten betrachtet. Diskursstränge können Jäger zufolge synchron oder diachron analysiert werden, das heißt gegenwartsbezogen oder über einen längeren historischen Zeitraum hinweg. ${ }^{58}$ Nachdem es in der vorliegenden Untersuchung um die Frage geht, wie in aktuellen queeren

R. Diaz-Bone: Kritische Diskursanalyse: Zur Ausarbeitung einer problembezogenen Diskursanalyse im Anschluss an Foucault. Siegfried Jäger im Cespräch mit Rainer Diaz-Bone.

52 S. Jäger: Kritische Diskursanalyse, S. 26.

53 S. van Dyk: Verknüpfte Welt oder Foucault meets Latour. Zum Dispositiv als Assoziation.

54 S. Jäger: Kritische Diskursanalyse, S. 113.

55 Ebd., S. 38.

56 Daniel Bartel/Peter Ullrich/Kornelia Ehrlich: »Kritische Diskursanalyse: Darstellung anhand der Analyse der Nahostberichterstattung linker Medien«, in: Ulrike Freikamp/Matthias Leanza/Janne Mende et al. (Hg.), Kritik mit Methode? Forschungsmethoden und Cesellschaftskritik, Berlin: Karl Dietz Verlag 2008, S. 53-73, hier S. 59. 
Diskursen, vor dem Hintergrund einer queeren Kritik an eindeutigen Identitätspolitiken, ausgehandelt wird, wer das queere politische Subjekt ist oder sein will, wurden entsprechend aktuelle queer-aktivistische Diskurse - in ihrer synchronen Dimension analysiert. ${ }^{59}$ Die Auswahl der Projekte, deren diskursive Aushandlungsprozesse analysiert wurden, ist bereits im vorherigen Kapitel ausführlich beschrieben worden. Das gesamte Text- und Bildmaterial, das die ausgewählten queeren Einrichtungen produzieren, wurde zudem noch zeitlich eingrenzt. Hierzu war der Begriff des diskursiven Ereignisses von Jäger hilfreich: »Als diskursive Ereignisse sind nur solche im Diskurs angesprochene Ereignisse zu fassen, die medial groß herausgestellt werden und als solche medial groß herausgestellten Ereignisse die Richtung und die Qualität des Diskursstrangs, zu dem sie gehören, beeinflussen oder wesentlich bestimmen. ${ }^{60}$ Ausgehend von diesem Verständnis eines diskursiven Ereignisses, ist auch der Analysezeitraum der vorliegenden Untersuchung eingegrenzt worden. Dieser beginnt 2010 mit der Ablehnung des Preises für Zivilcourage des Berliner CSD durch Judith Butler, die als ein diskursives Ereignis in der queeren Community im deutschsprachigen Raum verstanden werden kann. Als Ende des Analysezeitraums wurde das Jahr 2016 gewählt. Dieser Entscheidung lag zunächst die pragmatische Notwendigkeit, das Material zu begrenzen, zugrunde. Es hat sich aber gezeigt, dass auch dem Ende des Analysezeitraums ein diskursives Ereignis folgt. Zumindest in Deutschland - aber auch darüber hinaus im deutschsprachigen Raum rezipiert - hat es Anfang 2017 mit dem Erscheinen von »Beißreflexe « und den daran anschließenden Diskussionen ein weiteres zentrales diskursives Ereignis in der queeren Community gegeben. ${ }^{61}$ Daher kann der Analysezeitraum - 2010 bis 2016 - zwischen zwei diskursiven Ereignissen verortet werden.

Nachdem in der ersten Phase der Untersuchungsgegenstand bestimmt wird, wird in der zweiten Phase - der Erhebungsphase - das Archiv erstellt. Das bedeutet, es wird das gesamte in diesem Zeitraum produzierte Text- und Bildmaterial gesammelt. ${ }^{62}$ Die Beschreibung der Archiverstellung folgt in Kapitel sechs.

Der dritte Schritt - die Strukturanalyse - stellt so etwas wie das »Herzstück der Diskursanalyse « dar. Da es aufgrund der Materialfülle unmöglich ist, alle Diskursfragmente im Detail zu analysieren, geht die Strukturanalyse der Feinanalyse voraus. Die Strukturanalyse dient dazu, einen Überblick über die Diskurse zu bekommen und unterscheiden zu können, welche »Aussagen « »belanglose und eher oberflächliche Äußerungen « sind und welche in einem bestimmten Diskurs "Gewicht haben«. Entsprechend wird zunächst einmal das gesamte Text- und Bildmaterial strukturanalytisch aufgearbeitet. ${ }^{63}$ Die Strukturanalyse dient dann dazu, repräsentative Diskursfragmente das sind einzelne Texte oder Textteile, die in ihrer Gesamtheit einen Diskursstrang bilden - für die im vierten Schritt folgende Feinanalyse begründet auszuwählen: »Erst im

59 Da nicht alle Diskursfragmente analysiert werden konnten, wurde auch hier eine Auswahl getroffen, die in Kapitel sechs dargelegt wird.

60 S. Jäger: Kritische Diskursanalyse, S. 82.

61 Vgl. J. Degeling/S. Horn: ২Queer aufs Spiel gesetzt. Über Beißreflexe, queere Bewegungsgeschichte und gegenwärtige Affektkulturen.

62 Die Beschreibung des »Archivs« folgt in Kapitel sechs.

63 S. Jäger: Kritische Diskursanalyse, S. 96. 
Zusammenhang von Struktur- und Feinanalyse(n) lässt sich der betreffende Diskursstrang als Ganzer einschätzen. «" ${ }^{64}$ Aus den von Jäger vorgeschlagenen Strukturmerkmalen wurden für diese Arbeit folgende Strukturmerkmale für das gesamte in das Archiv aufgenommene Material herausgearbeitet: Datum, Titel, Textsorte, Autor*innen, Überschrift des Artikels, Anlass des Artikels, formale Besonderheiten (Grafik, Überschrift), Themen/Unterthemen, Aussagen, Quellen des Wissens, Normalismen, Auffälligkeiten. Die Strukturanalyse findet auf verschiedenen Ebenen Eingang in die Arbeit, oft ohne explizit benannt zu werden. Zunächst einmal hat sie die Beschreibung der Projekte informiert. Auch die konkrete Beschreibung des Archivs - der erste Einblick in das von den einzelnen Projekten produzierte Text- und Bildmaterial - in Kapitel sechs geht auf die Erkenntnisse der Strukturanalyse zurück. Entsprechend der primären Funktion der Strukturanalyse, wurde die Auswahl der repräsentativen Diskursfragmente mit den strukturanalytischen Erkenntnissen begründet und auch die Feinanalyse ist durch das in der Strukturanalyse erarbeitete Wissen informiert.

Die von Jäger für den vierten Schritt - die Feinanalyse - vorgeschlagene Herangehensweise versteht er als eine »Werkzeugkiste«, derer mensch sich bedienen kann und die entsprechend auch in dieser Arbeit innerhalb geringer Abweichungen, manchmal auch von Diskursfragment zu Diskursfragment, unterschiedlich genutzt wurde. Die Feinanalyse umfasst vier Schritte: die Analyse des »institutionellen Rahmens«, der »TextOberfläche«, der »sprachlich-rhetorischen Mittel« und der »inhaltlich-ideologischen« Aussagen. Der erste Schritt der Feinanalyse ist die Analyse des institutionellen Rahmens. Das beinhaltet zunächst einmal eine »allgemeine Charakterisierung der Zeitschrift oder Zeitung bzw. des Genres etc. « und die »Bestimmung der Textsorte $«{ }^{65}$ Diese beiden Aspekte sind in Kapitel sechs ausführlich ausgearbeitet. Hier werden neben der Beschreibung des Archivs auch die analysierten Medien genrespezifisch eingeordnet. Darüber hinaus umfasst die Analyse des »institutionellen Rahmens« die Fragen, wie das Thema an anderer Stelle verhandelt worden ist und welche Rolle die Autor*innen beziehungsweise deren Diskursposition für die Texte spielen. ${ }^{66}$ Diese Aspekte wurden, je nach Relevanz, für die Feinanalyse herausgearbeitet und werden zumeist zu Beginn der Feinanalyse dargestellt. Der Analyse des institutionellen Rahmens folgt nach Jäger, zweitens, die Analyse der »Text-Oberfläche«. Bei diesem Analyseschritt geht es zunächst einmal darum, den Inhalt des Textes und der einzelnen Abschnitte zu erfassen, aber auch einen Blick auf die "graphische« Darstellung und deren Funktion im Text zu werfen. ${ }^{67}$ In einem dritten Schritt werden dann die "sprachlich-rhetorischen « Mittel der Diskursfragmente analysiert. Es wird beispielsweise auf die Übergänge in den einzelnen Abschnitten geachtet, zudem werden die Argumentationsstrategie sowie die Verwendung von bestimmten »sprachliche[n] Routinen «, »Kollektivsymbolen«, »Metaphern « oder anderen Sprachbildern und die Funktion der Verwendung bestimmter Modi, Tempi oder Pronomen herausgearbeitet. ${ }^{68}$ In einem vierten Schritt werden

\footnotetext{
64 Ebd., S. 97.

65 Ebd., S. 99.

66 Ebd., S. $100 f$.

67 Ebd., S. 102.

68 Ebd., S. 103-107.
} 
dann die »inhaltlich-ideologischen « Aussagen bestimmt. ${ }^{69}$ Auf einer inhaltlichen Ebene wurden die Diskursfragmente vor allem im Hinblick auf die Fragestellung dieser Arbeit betrachtet. Abschließend wird der Bezug zum »diskursiven Kontext« hergestellt. Das passiert in der vorliegenden Arbeit auf mehreren Ebenen: Zunächst einmal können die theoretischen wie bewegungsgeschichtlichen Auseinandersetzungen auch als Teil des diskursiven Kontextes, in dem die jeweiligen Debatten der ausgewählten Diskursfragmente stattfinden, verstanden werden. In den Diskursen der Projekte wird oft explizit oder implizit auf die in den ersten drei Kapiteln der vorliegenden Arbeit dargestellten theoretischen Überlegungen Bezug genommen. Die jeweils für die Feinanalyse ausgewählten repräsentativen Themen (Pride, Mehrfachdiskriminierung und Comingout) wurden selbst noch einmal in einem spezifischeren diskursiven Kontext verortet. Auch lokal geführte Debatten in der Community stellen einen Aspekt des diskursiven Kontextes dar, in den die Diskursfragmente eingeordnet wurden. Auch wenn diese Analyseschritte in der Darstellung nicht immer getrennt voneinander auftreten und der Bezug zu Jäger nicht immer explizit benannt wird, so ist es doch seine »Werkzeugkiste«, aber auch das Verständnis, das Foucaults eigene Analysen vermittelt haben, die im Hintergrund der Analyse steht. Nachdem der diskursanalytische Zugang der Arbeit nun umrissen wurde, folgt die konkrete Begründung der Auswahl des Untersuchungsgegenstandes - das, was Jäger in die »Konzeptions- und Archiverstellungsphase« einordnen würde. 
